Annuaire suisse de politique de développement

18 | 1999

La Suisse et l'action humanitaire

\title{
3. Environnement et développement
}

Catherine Schümperli Younossian et Marc Hufty

\section{OpenEdition}

\section{Journals}

Édition électronique

URL : http://journals.openedition.org/aspd/681

DOI : 10.4000/aspd.681

ISSN : 1663-9669

\section{Éditeur}

Institut de hautes études internationales et du développement

\section{Édition imprimée}

Date de publication : 1 janvier 1999

Pagination : 141-154

ISSN : 1660-5934

\section{Référence électronique}

Catherine Schümperli Younossian et Marc Hufty, « 3. Environnement et développement », Annuaire suisse de politique de développement [En ligne], 18 | 1999, mis en ligne le 14 juillet 2012, consulté le 08 septembre 2020. URL : http://journals.openedition.org/aspd/681; DOI : https://doi.org/10.4000/aspd. 681 


\subsection{SUIVI DE LA CONFÉRENCE DE RIO}

En 1998, l'environnement de la planète aura connu de nombreuses catastrophes écologiques dévastatrices: incendies de forêts incontrôlables en Indonésie, inondations destructrices en Chine, ouragan mortel en Amérique centrale. Comme chaque année, les Etats se sont réunis à New York pour analyser la mise en ceuvre de l'Agenda 21 lors de la $6^{e}$ Commission pour le développement durable. En Suisse, le Conseil pour le développement durable a commencé ses travaux et le Conseil fédéral a adopté les lignes directrices pour la politique internationale de la Suisse en matière de développement durable. La campagne Nord/Sud pour un développement durable des ouvres d'entraide suisses s'est poursuivie en 1998.

\section{$\square$ Commission pour le développement durable (CDD-6)}

La Commission pour le développement durable a été créée à la suite de la Conférence de Rio pour contrôler et encourager la mise en ouvre des chapitres de l'Agenda 21. Depuis 1993, elle se réunit chaque année afin de discuter cette mise en ouvre et de donner des recommandations pour les travaux ultérieurs au niveau national et international. La CDD ne peut pas prendre des décisions juridiquement contraignantes, mais par ses travaux elle a déclenché de nombreuses initiatives bilatérales et multilatérales et a contribué à une meilleure coordination des activités du suivi de Rio au sein du système onusien.

La Commission pour le développement durable s'est réunie pour la sixième fois (CDD-6) à New York durant deux semaines (du 20 avril au $1^{\text {er }}$ mai 1998). Les participants ont travaillé dans différents comités de rédaction chargés de négocier des projets de décisions qui sont ensuite approuvés en séance plénière. La délégation suisse, conduite par le secrétaire d'Etat Franz Blankart, de l'OFAEE, était composée de représentants de son office ainsi que de l'OFEFP, de la DDC et de l'OFDE. Les ONG étaient représentées par la Communauté de travail.

Les thèmes principaux abordés lors de cette réunion ont été l'industrie et le développement durable, la définition d'une stratégie pour la gestion de l'eau douce et la mise en œuvre d'un programme d'action pour les «petites îles des pays en développement». Un troisième groupe de travail s'est penché sur les thèmes intersectoriels suivants: transferts de technologies, renforcement des capacités, éducation et «conscientisation» du public, science pour le développement durable. Les trois derniers jours de la session ont été consacrés au segment ministériel ${ }^{2}$.

* Parties 3.1, 3.2 et 3.3: par Catherine Schümperli Younossian, chargée de recherche à l'IUED.

Partie 3.4: par Marc Hufty, chargé de cours à l'IUED.

1. Le Worldwatch Institute de Washington publie chaque année un état des lieux de l'environnement. Le rapport est intitulé Worldwatch Database 1998, Worldwatch Institute, Washington, May 1998. Site Internet: http://www.worldwatch.org/

2. Le résumé complet ainsi que les principales décisions de la CDD-6 se trouvent sur le site du Earth Negociation Bulletin à la page http://www.iisd.ca/linkages/csd/csd6.html 


\section{Eau douce}

Les décisions concernant le thème de l'eau douce contiennent entre autres des recommandations sur les transferts de technologies, les ressources et les mécanismes de financement, l'amélioration de la collecte de données scientifiques, la protection des écosystèmes comme réserve naturelle d'eau douce, l'amélioration des capacités nationales pour l'approvisionnement des populations en eau potable. Les délégués ont notamment travaillé sur un Programme d'actions prioritaires et sur une déclaration ministérielle adoptés lors de la Conférence internationale sur l'eau et le développement durable, qui s'est tenue à Paris du 19 au 21 mars $1998^{3}$.

La contribution de la Suisse à la question de l'eau douce s'est faite par la présentation d'une publication intitulée Mountains of the World: Water Towers for the 21st Century ${ }^{4}$ Editée par l'Institut de géographie de 1'Université de Berne, avec le soutien de la DDC, cette brochure démontre l'importance des territoires de montagne dans l'approvisionnement en eau. Elle contient 12 cas concrets de relation entre un environnement de montagne et le bassin fluvial qu'il alimente.

\section{Industrie et développement durable}

Par essence le thème «industrie et développement durable» est un thème difficile et conflictuel entre les pays industrialisés et les pays du Sud. Représentants gouvernementaux, industriels, syndicats et ONG ont débattu sur quatre points particuliers: la responsabilité des entreprises, les outils de gestion environnementale et sociale, la coopération technologique, l'utilisation de l'eau douce par l'industrie. Le texte adopté reflète les positions des uns et des autres. Les priorités de la Suisse y sont reflétées notamment en ce qui concerne le respect des normes du travail et de l'environnement, la conclusion d'accords volontaires avec le secteur industriel, le soutien des PME et l'encouragement à la recherche. Il est intéressant de constater que la CDD s'ouvre plus aux questions sociales.

\section{Réunion de haut niveau}

Les trois derniers jours de la CDD furent consacrés au segment ministériel lors duquel chefs de gouvernement et hauts fonctionnaires ont communiqué les déclarations de leurs Etats. Les pays du Sud ont particulièrement insisté sur la difficulté à financer la mise en œuvre de l'Agenda 21 et sur l'importance de l'engagement des pays industrialisés dans ce domaine. Mais la plupart des déclarations se concentrèrent sur les thèmes de la commission. La déclaration de la Suisse a été présentée par le secrétaire d'Etat Franz Blankart. Après avoir relevé la nécessité d'une gestion durable des ressources en eau, F. Blankart a surtout insisté sur la responsabilité de l'industrie en matière environnementale et sociale et s'est réjoui du rôle actif que celle-ci a joué lors de la Commission pour le développement durable. Il a également rappelé l'importance du respect des normes fondamentales du travail, comme garant de la qualité de l'emploi, de l'environnement et de la productivité. Et de préciser à l'égard des pays en développement: «Si nous proposons l'inclusion, dans certaines conventions internationales, des

3. Parrainée par le gouvernement français, la conférence a réuni plus de 600 participants incluant ministres et hauts responsables de la gestion de l'eau venus de 84 pays, ainsi que des représentants de la société civile, des agences des Nations Unies, des organisations internationales et des banques de développement impliqués dans le secteur de l'eau. Le compte-rendu complet de la Conférence de Paris se trouve sur le site du Bulletin des négociations de la Terre http://www.iisd.ca/linkages/french/

4. A commander auprès de Mountain Agenda, c/o Institut de géographie de l'Université de Berne, Hallerstrasse 12, CH3012 Berne. 
droits sociaux et des normes environnementales, il ne faut pas nous soupçonner d'une motivation protectionniste.» Enfin, le secrétaire d'Etat a relevé le rôle essentiel que doit jouer l'Etat dans la formulation d'une politique sociale et environnementale adéquate, citant en exemple la réforme fiscale sur laquelle la Suisse travaille qui vise à taxer l'utilisation de ressources non renouvelables plutôt que le travail.

\section{$\square$ Suivi de Rio en Suisse}

\section{Conseil pour le développement durable}

Le 25 février 1998, le Conseil fédéral a institué le Conseil pour le développement durable, un organe consultatif destiné à orienter le Conseil fédéral et les départements en matière de développement durable. Ce conseil sera également appelé à formuler des propositions encourageant une réorientation de la société vers le développement durable. Il devra en outre évaluer certains aspects de la politique fédérale sous cet angle.

Le Conseil pour le développement durable compte 13 membres qui exercent leur mandat à titre personnel et indépendant. Sa présidence a été confiée à Anne Petitpierre, professeure de droit à 1'Université de Genève. Le conseil s'est réuni à trois reprises en 1998 et a défini les thèmes de travail pour les mois à venir: politique énergétique en Suisse, définition d'indicateurs, réflexion sur l'opportunité d'une réforme institutionnelle visant de meilleurs mécanismes de décision en matière de développement durable.

Campagne Nord/Sud pour un développement durable de la Communauté de travail Lancée en janvier 1997, la campagne Nord/Sud pour le développement durable s'est poursuivie en 1998. Le point fort de la campagne en 1998 a été une importante conférence qui s'est tenue durant une semaine au Palais fédéral, à Berne, en mai 1998. Plus de 800 participants, dont une centaine venus de 40 pays en développement, ont réfléchi sur des thèmes Nord-Sud ${ }^{5}$. Les exposés et les résumés des différents ateliers ont fait l'objet d'une publication ${ }^{6}$.

\section{Examen des performances environnementales de la Suisse par l'OCDE}

L'Organisation de coopération et de développement économiques (OCDE) examine depuis 1992 les performances environnementales de ses pays membres. Pour la première fois, la Suisse a fait l'objet de ce type d'étude, qui ne vise pas à dresser un état des lieux de l'environnement, mais plutôt à évaluer l'efficacité des mesures politiques qui s'y rapportent. L'OCDE confirme les succès de la Suisse notamment dans les domaines de la protection de l'air et des eaux. Elle relève également la bonne politique des transports menée par le gouvernement. Toutefois, elle constate une forte diminution des espaces naturels et, parallèlement, une réduction de la diversité biologique. Elle constate enfin que le budget environnemental de la coopération au développement, relativement modeste, devrait être augmenté .

5. La Communauté de travail a créé une page Internet qui résume les principales interventions et conclusions de cette conférence: http://www.swisscoalition.ch/seiten.f/4Ce/4Ce.htm

6. Actes de la Conférence sur le développement durable, à commander auprès de la Communauté de travail, Berne.

7. OCDE, Examens des performances environnementales, Suisse, OCDE, Paris, 1998, 244 p. 
SOURCES

Office fédéral de l'environnement, des forêts et du paysage (OFEFP), Communiqués de presse 1998, disponibles sur Internet.

Arthur Mohr, «Nachhaltige Entwicklung, Umweltschutz und ökonomische Instrumente: Umsetzung in der Schweiz», Schweizerische Zeitschrift für Volkswirtschaft und Statistik, Helbing \& Lichtenhahn, 3.9.98, S. 317 und f.

Lucas Bretschger, «Nachhaltige Entwicklung der Weltwirtschaft: Ein Nord-Süd Ansatz», Schweizerische Zeitschrift für Volkswirtschaft und Statistik, Helbing \& Lichtenhahn, 3.9.98, S. 369 und f.

SITES INTERNET

OFEFP: http://www.admin.ch/buwal/f/index.htm

Communauté de travail: http://www.swisscoalition.ch/

The Earth Negotiation Bulletin propose des résumés détaillés sur les principaux pourparlers en matière d'environnement et de développement aux Nations Unies: http://www.iisd.ca/linkages/ Il existe également une version française, le Bulletin des négociations de la Terre, qui traduit certains des documents.

\subsection{FONDS POUR L'ENVIRONNEMENT MONDIAL}

Le Fonds pour l'environnement mondial est un mécanisme financier visant à soutenir des projets environnementaux de portée globale. Créé en 1990, le FEM a été restructuré en 1994 et réapprovisionné à deux reprises (1994 et 1998). La structure institutionnelle du FEM repose sur trois organisations internationales (Banque mondiale, PNUD et PNUE). La $1^{r e}$ Assemblée générale du FEM s'est tenue à New Delhi en avril 1998. La Suisse lui accorde une attention particulière et est membre de son Conseil exécutif. Lors de la session d'été 1998, le Parlement a accepté un nouveau crédit-cadre de 88,5 millions de francs pour la participation de la Suisse au deuxième réapprovisionnement du FEM (1998-2000) et pour le fonds multilatéral du Protocole de Montréal.

\section{$\square$ Objectifs du FEM}

Le Fonds pour l'environnement mondial (FEM) est un mécanisme de financement multilatéral qui accorde des contributions non remboursables aux pays en développement et aux pays d'Europe centrale et orientale pour des projets environnementaux de portée globale. Ses activités sont menées conjointement par trois organisations internationales: la Banque mondiale, le Programme des Nations Unies pour le développement (PNUD) et le Programme des Nations Unies pour l'environnement (PNUE). Sa tâche principale consiste à mettre à la disposition des pays en développement des moyens permettant le financement de la mise en œuvre des conventions sur les changements climatiques et sur la diversité biologique, ainsi qu'à soutenir des projets concernant les eaux internationales et la couche d'ozone. Le FEM couvre également des domaines directement liés aux précédents, comme la protection des forêts, la remise en état d'écosystèmes endommagés ou la réduction des rejets de polluants et de déchets dans les eaux.

\section{$\square$ Restructuration et reconstitution du FEM}

L'idée du Fonds pour l'environnement mondial a été lancée par la France et l'Allemagne en 1989. Les deux pays avaient décidé de mettre chacun environ 140 millions de dollars américains à condition qu'un nombre suffisant d'autres pays donateurs y participent également. En 1990, la décision était prise d'entamer une phase pilote (1991-1993). La Suisse a contribué au fonds central du FEM à raison de 40 millions de dollars. En outre, elle a mis à disposition 14 millions de 
dollars supplémentaires sous forme de cofinancement de projets sélectionnés. Le FEM disposait alors d'un montant de 800 millions de dollars pour une phase expérimentale de trois ans.

Après cette phase, des négociations ont eu lieu, visant la restructuration et le réapprovisionnement du Fonds pour la période 1994-1997 (FEM-1). Cette restructuration du FEM a permis de prendre en considération certaines critiques formulées lors de la phase pilote. Une représentation plus équilibrée entre les pays industrialisés et les pays en développement, la révision du mode de scrutin (voir ci-dessous) et une plus grande transparence dans la conduite des affaires ont ainsi été prises en compte. Le FEM a également été désigné comme mécanisme de financement provisoire des conventions sur les changements climatiques et la diversité biologique. Les négociations sur le réapprovisionnement du Fonds ont été menées parallèlement et ont abouti à une dotation de 2 milliards de dollars. La barre des 3 milliards de dollars, objectif avoué de nombreux Etats européens dont la Suisse, n'a pas été atteinte. Pour sa part, la Suisse a contribué au réapprovisionnement du fonds pour un montant de 65 millions de francs suisses. Par cette contribution, elle s'assure un siège au sein du Conseil du FEM. En avril 1998, 164 pays étaient membres du FEM.

Pour sa deuxième reconstitution (période 1998-2002), le FEM a été doté par les pays donateurs d'un financement de 2,75 milliards de dollars. La Suisse a participé pour un montant de 70 millions de francs (voir ci-dessous). Les fonds sont versés sous forme de contributions non remboursables et sont destinés en priorité à financer les coûts excédentaires qu'occasionne le fait de conférer un aspect environnemental de portée globale à un projet existant. Mais le FEM finance également des projets dits indépendants, qui ne pourraient être réalisés sans son soutien. La plupart des «projets indépendants» ont un caractère innovateur dont la valeur économique est difficile à chiffrer. Les ressources servant à financer ces projets sont souvent complétées par des contributions d'organisations environnementales. En principe tous les Etats dont le revenu annuel par habitant est inférieur à 4000 dollars peuvent prétendre à une aide du FEM. Afin de soutenir également des initiatives plus modestes, le FEM a créé un programme de microfinancement. Ce programme est géré par le PNUD et accorde des contributions d'un montant maximum de 50'000 dollars à des actions locales, dont bénéficient principalement de petites organisations non gouvernementales.

\section{$\square$ Structure institutionnelle du FEM}

L'identification, la préparation et l'exécution des projets du FEM reposent sur trois organisations internationales existantes: la Banque mondiale (gestion des fonds, prise en charge des projets d'investissements), le PNUD (chargé des projets de renforcement des capacités institutionnelles, coopération technique et formation), le PNUE (exécute des projets globaux de formation et d'information dans les quatre domaines d'intervention prioritaires du Fonds et dirige le STAP ${ }^{8}$ ).

Les directives stratégiques et programmatiques du FEM sont définies par le Conseil, constitué de 32 membres (dont 16 représentent les pays du Sud, 2 les pays de l'Europe centrale et orientale et 14 les pays industrialisés), qui se réunit deux fois par an. La structure décisionnelle du Conseil se distingue par une

8. Le STAP est le groupe consultatif pour la science et la technologie du FEM. Organe indépendant, il fournit des conseils d'ordre scientifique et technique. 
caractéristique intéressante. Très critiqué par les pays en développement lors de la phase pilote et suite à une évaluation indépendante, le système de décision a été revu lors de la première restructuration du FEM en 1994. Dans la mesure du possible, les décisions du Conseil se font de manière consensuelle. Lorsque cela n'est pas possible, la décision est prise sur un mode de scrutin à double majorité, qui requiert les voix de $60 \%$ de tous les Etats membres du FEM, ainsi que l'aval de pays donateurs représentant au moins $60 \%$ des contributions totales. Cette structure décisionnelle constitue une nouveauté dans le cadre institutionnel. Elle associe les mécanismes décisionnels des banques multilatérales de développement, où les contributions financières déterminent le nombre de voix, et des organisations des Nations Unies, où l'on applique le principe «un pays - une voix».

L'Assemblée, composée de tous les Etats membres, a pour tâche d'examiner la politique générale du Fonds. L'Assemblée se réunit tous les trois ans. La première réunion s'est tenue en avril 1998 à New Delhi.

Enfin, le Secrétariat, dont le siège est à Washington, a pour fonction d'assurer l'application des décisions de l'Assemblée et du Conseil, de coordonner la formulation du programme d'action du FEM et d'en surveiller l'exécution. Il doit également veiller à la mise en œuvre des politiques opérationnelles adoptées par le Conseil.

\section{$\square 1^{\text {re }}$ Assemblée du FEM}

La $1^{\text {re }}$ Assemblée générale du Fonds pour l'environnement mondial s'est tenue à New Delhi (Inde), du $1^{\text {er }}$ au 3 avril 1998. Elle a réuni plus de 900 personnes: les représentants des délégations officielles des 164 pays membres, d'organisations internationales et non gouvernementales, ainsi qu'un panel de scientifiques et des journalistes. Les deux jours précédant l'ouverture de l'Assemblée ont permis au Conseil exécutif de se réunir pour une de ses deux réunions annuelles. Lors de cette réunion, le Conseil a notamment approuvé le budget pour l'année 1999, mais s'est déclaré inquiet du rythme de croissance des dépenses et des frais généraux liés à l'exécution des projets. Il a demandé que des mesures soient prises pour réduire les frais administratifs.

Cette assemblée était la première occasion pour tous les membres du FEM d'échanger leurs points de vue sur la politique et les opérations du Fonds. Quatre rapports ${ }^{9}$ ont été soumis à l'Assemblée. Le plus attendu, Bilan global du fonctionnement du FEM (en anglais Study of the GEF's Overall Performance), fait l'objet d'une importante discussion. Il relève notamment que le FEM n'a pas été capable d'intégrer suffisamment les préoccupations environnementales dans les opérations régulières des principales agences de mise en œuvre (BM, PNUE, PNUD). Il souligne également que la transparence du FEM à l'égard des ONG et du secteur privé doit encore être améliorée. Pour pallier les principales critiques, des recommandations ont été proposées, auxquelles le Secrétariat du FEM devra donner suite. En marge des séances plénières se sont tenus un certain nombre de panels de discussion, ateliers de travail et séminaires ${ }^{10}$.

9. Bilan global du fonctionnement du FEM, Etude des enseignements des projets du FEM, Examen de l'état d'avancement des projets du FEM, Rapport du directeur général sur les politiques, les opérations et l'avenir du FEM.

10. http://www.iisd.ca/linkages/sd/gefasmbly.html pour consulter les comptes-rendus de ces diverses rencontres sur Internet. 
A l'issue de la $1^{\text {re }}$ Assemblée du FEM, les 164 Etats membres ont adopté la «Déclaration de New Delhi», qui confirme le FEM dans son rôle de mécanisme de financement multilatéral de protection de l'environnement mondial, en soulignant son importance dans la réalisation des objectifs des conventions et des protocoles environnementaux (conventions sur la biodiversité et le climat, protocoles de Kyoto et de Montréal, etc.). La Déclaration se félicite également de la deuxième reconstitution des ressources du FEM à hauteur de 2,75 milliards de dollars. Enfin, le texte énumère 12 mesures qui doivent permettre au FEM d'améliorer son efficacité pour accomplir ses multiples missions.

\section{Position de la Suisse}

Depuis la création du FEM, la Suisse a participé activement à son élaboration, sa restructuration et son financement. Le montant de ses contributions financières lui vaut un siège (occupé par Philippe Roch, directeur de l'OFEFP) au sein du Conseil exécutif, position qui lui permet d'influencer directement la politique du FEM.

La position de la Suisse a été présentée par Philippe Roch. Le directeur de l'Office fédéral de l'environnement, des forêts et du paysage a tout d'abord rappelé que la Suisse souhaite que le FEM soit désigné comme mécanisme de financement permanent des conventions sur le climat et la biodiversité, ainsi que des différents protocoles environnementaux (Kyoto, Montréal, etc.). Il a également souligné le rôle du FEM pour une meilleure prise en compte des préoccupations environnementales auprès des agences d'exécution (BM, PNUD et PNUE). Enfin il a rappelé qu'il faut développer des indicateurs et des méthodes de travail se prêtant à l'analyse et à l'évaluation régulière afin d'augmenter l'efficacité et la flexibilité du FEM.

La Suisse attache une attention particulière à la mise en œuvre des recommandations du rapport Bilan global du fonctionnement du FEM et c'est pourquoi elle a créé un groupe de travail conjoint DDC-OFEFP, intitulé Réseau d'appui, chargé de formuler des propositions concrètes pour cette mise en œuvre. Le Réseau d'appui prend également position sur les projets soutenus par le FEM (commentaires techniques).

\section{FEM et $O N G$}

Depuis sa création, les ONG ont formulé de nombreuses critiques à l'égard du FEM. Tout en lui reconnaissant un important potentiel, financier et opérationnel, elles considèrent que les résultats tardent à venir. Leurs principaux doutes concernent la lourdeur institutionnelle du FEM, son manque de transparence et la nécessité de mettre en place un système efficace d'évaluation et de monitoring de l'utilisation des ressources du Fonds. Elles soulignent également le manque de progression dans l'effort d'intégration des objectifs environnementaux au sein des différentes agences chargées de la mise en œuvre du FEM. Enfin, elles souhaiteraient être mieux impliquées dans les différentes étapes du projet, de l'identification à sa réalisation. Présentes lors de la $1^{\text {re }}$ Assemblée du FEM, elles ont réitéré leurs critiques.

Pourtant, les ONG sont maintenant plus de 200 accréditées auprès du Fonds. Celui-ci collabore avec elles en leur confiant l'exécution de certaines composantes de projets. Souvent, elles jouent un rôle important dans l'identification et la préparation de ces projets. Outre ce travail sur le plan opérationnel, les ONG 
s'avèrent également des partenaires importants sur le plan politique. Elles ont notamment participé aux travaux de réflexion précédant la restructuration du Fonds. D'autre part, avant chaque réunion du Conseil, des consultations avec des ONG ont lieu.

La Communauté des œuvres d'entraide a pris position à diverses reprises (Prise de position de la politique de développement 1997) pour demander une évaluation complète des activités du Fonds, ainsi que des résultats obtenus dans les projets soutenus. Elle insiste également sur le fait que ces études doivent être rendues publiques.

En outre, l'UICN a publié une brochure intéressante ${ }^{11}$ à l'attention des ONG. Ce «guide» cherche à stimuler l'intérêt et l'engagement des ONG auprès du FEM dans les domaines de politique générale, d'évaluation et de monitoring, et enfin dans la mise en œuvre de projets.

\section{$\square$ Crédits-cadres de la Suisse en faveur de l'environnement global}

Dans le cadre du $700^{\mathrm{e}}$ anniversaire de la Confédération (1991), le Parlement a accordé un crédit-cadre de 300 millions de francs pour le financement, dans les pays en développement, de programmes et de projets en faveur de l'environnement global; 120 millions ont été attribués à des fonds multilatéraux (Fonds ozone découlant du Protocole de Montréal et Fonds pour l'environnement mondial) et 180 millions à la mise en œuvre de mesures bilatérales dans les PVD.

Faisant suite au crédit-cadre de 1991 , dans un message ${ }^{12}$ concernant les fonds pour l'environnement mondial, le Conseil fédéral a demandé au Parlement la nouvelle mise à disposition de 90 millions de francs qui permettront à la Suisse de poursuivre son engagement pour la période de 1998 à 2002. Le 10 juin 1998, le Parlement a accordé un crédit de 88,5 millions de francs.

\section{SOURCES}

«Message concernant un crédit-cadre pour le financement de programmes et de projets environnementaux d'importance mondiale dans les pays en développement», 26 novembre 1997, message 97.083.

OFEFP, «FEM: penser et agir globalement», Environnement. Bulletin de l'OFEFP, 2/98.

Communauté de travail des œuvres d'entraide, Prise de position de la politique de développement 1997.

A. Steiner, S.W. Burgiel, S. Cohen, The Global Environment Facility. From Rio to New Delhi: A Guide for NGOs, IUCN, Gland (Switzerland) and Cambridge (UK), 1997.

SITES INTERNET

Site officiel du Fonds pour l'environnement mondial: http://www.gefweb.org/

Site de la Banque mondiale, contenant le compte-rendu du Conseil exécutif du FEM: http://www.worldbank.org/

11. A. Steiner, S.W. Burgiel, S. Cohen, The Global Environment Facility. From Rio to New Delhi: A Guide for NGOs, IUCN, Gland (Switzerland) and Cambridge (UK), 1997.

12. «Message concernant un crédit-cadre pour le financement de programmes et de projets environnementaux d'importance mondiale dans les pays en développement», 26 novembre 1997, message 97.083. 


\subsection{CONVENTION DE BÂLE SUR LES DÉCHETS DANGEREUX}

Suite à divers scandales liés à l'exportation de déchets dangereux vers les pays en développement, la communauté internationale a reconnu la nécessité de prendre des mesures pour régler cette question. Sous les auspices du PNUE, un instrument juridique de portée mondiale concernant la gestion écologiquement rationnelle des déchets dangereux, leurs mouvements transfrontières et leur élimination a été élaboré dans les années 80 . La Convention de Bâle, adoptée en 1989, compte 117 Etats membres, qui se sont réunis à quatre reprises pour organiser sa mise en œuvre. La Suisse a joué un rôle particulièrement actif dans son élaboration et Genève accueille son Secrétariat.

Plus de 400 millions de tonnes de déchets dangereux sont produites chaque année dans le monde. Quelque 10\% de ces déchets traversent les frontières. Stockage, acides corrosifs, produits chimiques organiques, métaux toxiques et autres déchets représentent une menace aiguë, et cela à long terme, pour la santé et pour l'environnement (contamination des nappes phréatiques, filtration, diverses pollutions). Pour des raisons économiques, un important volume de ces déchets fut transporté des pays industrialisés vers les pays en développement et les pays de l'Europe de l'Est, où les coûts d'élimination étaient moindres. Cependant, ces pays n'avaient pas le savoir-faire ni la capacité d'en assurer une gestion écologique. Aussi les mouvements transfrontières des déchets dangereux sont-ils devenus un problème mondial, auquel tente de répondre la Convention de Bâle.

\section{$\square$ Origines et objectifs de la Convention de Bâle}

La Convention de Bâle sur le contrôle des mouvements transfrontières de déchets dangereux et de leur élimination a été adoptée à Bâle en 1989 et est entrée en vigueur en 1992. Développée sous les auspices du Programme des Nations Unies pour l'environnement (PNUE), elle compte (selon un état de juin 1998) 117 Etats et la Communauté européenne; il est à noter que les Etats-Unis sont le seul pays occidental à n'avoir pas ratifié la Convention.

Le but général de la Convention de Bâle est de protéger la santé humaine et l'environnement contre les effets nuisibles qui peuvent résulter de la production, des mouvements transfrontières et de la gestion de déchets dangereux. Pour ce faire, un certain nombre d'objectifs doivent être atteints:

- réduction des mouvements transfrontières de déchets dangereux visés par la Convention afin d'atteindre un minimum compatible avec leur gestion écologique rationnelle;

- élimination des déchets dangereux et d'autres déchets, aussi près que possible de leur source de production;

- réduction de la production de déchets dangereux;

- strict contrôle des mouvements de déchets et prévention du trafic illicite;

- interdiction d'exportation de déchets dangereux vers des pays ne possédant pas de cadre juridique approprié ni les capacités techniques pour les gérer et les éliminer de manière écologiquement rationnelle;

口 aider les pays en développement et les pays à économie de transition à mettre en place une gestion écologique de leurs propres déchets. 
Quarante-cinq catégories de déchets sont concernées: rejets de produits pharmaceutiques et phytosanitaires, solvants, produits chlorés, résidus de sels de trempe, huiles minérales, polymères, métaux toxiques, colles et plastifiants, déchets contenant du cyanure, de l'amiante, etc. A noter que les déchets radioactifs ne sont pas compris dans le champs de la Convention de Bâle, mais sont soumis à d'autres instruments internationaux comme, par exemple, l'Agence internationale pour l'énergie atomique (AIEA).

Lors des négociations de la Convention, il est apparu qu'il serait trop facile à une partie de déroger à ses obligations en traitant avec un Etat non membre. Aussi un des principes essentiels de la Convention réside-t-il dans l'interdiction, pour les pays membres, d'exporter ou d'importer des déchets dangereux d'un Etat non partie.

\section{$\square$ La $4^{e}$ Conférence des parties à la Convention de Bâle (COP-4)}

La Conférence des parties, qui réunit les Etats ayant ratifié la Convention, est l'organe administratif de cette dernière. Sa principale fonction consiste à examiner et évaluer en permanence l'application de la Convention. Elle peut créer des organes subsidiaires jugés nécessaires à son application. Tout autre organe ou agence ayant des compétences dans le domaine des déchets dangereux (associations d'industries, ONG, OI) peut participer en tant qu'observateur à la Conférence.

Quatre réunions de la Conférence des parties ont eu lieu, en 1992, 1994, 1995 et 1998. La $1^{\text {re }}$ Conférence des parties demandait l'interdiction de l'exportation de déchets dangereux des pays industrialisés vers les pays en développement tandis que parallèlement les pays en développement s'engageaient à interdire l'importation de déchets illégaux et indésirables. Reconnaissant le désir croissant de la communauté internationale. d'interdire les mouvements transfrontières de déchets dangereux, et particulièrement les mouvements vers les pays en développement afin d'y éliminer les déchets, la seconde réunion des parties décida de l'interdiction immédiate de tous les mouvements depuis les pays de l'OCDE vers les pays non OCDE, de déchets dangereux destinés à une élimination finale. Par contre, les transports de déchets visant des opérations de recyclage ou de réutilisation ont été supprimés progressivement jusqu'au 31 décembre 1997, puis interdits à partir de cette date. Lors de la $3^{\mathrm{e}}$ Conférence des parties, le texte de la Convention fut amendé afin de tenir compte des décisions de la COP-2. La Conférence demanda également au groupe de travail technique de poursuivre son étude sur la caractérisation des déchets dangereux soumis à la Convention et de développer des directives techniques pour assister les Etats dans les mouvements transfrontières de déchets dangereux.

La $4^{\text {e }}$ Conférence des parties s'est tenue du 23 au 27 février 1998 à Kuching (Malaisie), en présence des représentants de 94 des 117 pays parties à la Convention. La délégation suisse était menée par Beat Nobs, chef de la division des Affaires internationales de l'OFEFP.

De nombreux points étaient à l'ordre du jour de la COP-4, dont le financement du Secrétariat, la mise en place d'un système d'échange d'informations sur les déchets et l'établissement de centres régionaux pour la formation et le transfert de technologie. Les deux décisions importantes concernent l'établissement de 
listes de déchets dangereux et l'amendement de l'Annexe VII (liste des pays exportateurs de déchets dangereux). Pour le premier point, la Conférence a adopté des listes de déchets afin de garantir les aspects juridiques de la mise en œuvre, dans les pays signataires, de l'interdiction d'exporter des déchets spéciaux. Par contre, pour le second, Israël, Monaco et la Slovénie, qui souhaitaient être retirés de la liste des pays exportateurs, se sont vu refuser cette demande. Lors du segment ministériel, de nombreux intervenants ont souligné la nécessité d'avoir un soutien technique en matière de gestion de déchets dangereux.

Position de la Suisse

La Suisse a activement participé à l'élaboration et à la conclusion de la Convention de Bâle. Depuis des années, elle soutient le Secrétariat de la Convention, basé à Genève. La Convention de Bâle reflète la politique menée par la Suisse en matière de déchets, puisqu'elle en interdit toute exportation. La Confédération s'est déclarée satisfaite des résultats de la COP-4, qui correspondent aux positions qu'elle y avait défendues. Lors de la conférence de Kuching, la Suisse s'est offerte à recevoir la $5^{\mathrm{C}}$ Conférence des parties à Bâle en décembre 1999, date qui marquera le $10^{e}$ anniversaire de la Convention. Y sera notamment discuté un protocole sur les responsabilités et les compensations pour les dommages résultant du commerce de déchets dangereux.

SOURCES

PNUE, Convention de Bâle, une solution mondiale pour contrôler les déchets dangereux, Nations Unies, New York et Genève, 1998.

Société pour la protection de l'environnement, Les déchets dangereux. Histoire, gestion et prévention, SPE et Georg Editeur, Genève, 1997.

«Basel Convention Parties to Examine Export Ban on Hazardous Wastes», Bridges, ICTSD, vol. 2, n 1 , JanuaryFebruary 1998.

SITE INTERNET

Site officiel de la Convention de Bâle: http://www.unep.ch/basel/index.html

ADRESSE UTILE

Secrétariat de la Convention de Bâle, Geneva Executive Center, 15, chemin des Anémones, 1219 Châtelaine (Genève). Tél. 022/917.82.18, fax 022/797.34.54.

\subsection{CONSERVATION DE LA DIVERSITÉ BIOLOGIQUE}

Six ans après la Conférence de Rio et l'élaboration de la Convention sur la diversité biologique, la diversité des gènes, des espèces, des écosytèmes dans le monde est toujours aussi menacée. Du fait de l'intensification de l'utilisation industrielle, agricole et pastorale des milieux naturels, des espèces s'éteignent quotidiennement, la pollution menace les écosystèmes fragilisés, les forêts brûlent et la désertification s'étend. La prise de conscience de la valeur intrinsèque ou économique des ressources biologiques a certes progressé, encore que ce soit difficile à mesurer avec exactitude, mais les moyens manquent. Faisant face à de multiples problèmes urgents, les pays du Sud ont peu de ressources à consacrer à la conservation et à la prévention à moyen et long terme. De plus, comme l'ont constaté divers forums internationaux, l'esprit de Rio est quelque peu retombé. Les intérêts divergents des différents acteurs, groupes de pays, organisations privées ou non gouvernementales, rendent difficile le maintien de la dynamique lancée à Rio et le passage à des actions concrètes.

ASTM 1997, pp. 55-59, «Mise en œuvre de la Convention sur la diversité biologique». 
Les Etats-Unis, acteur clé dans ce domaine du fait de l'importance des entreprises privées travaillant sur le vivant et des ONG environnementales, n'ont toujours pas ratifié la convention. Alors que la convention prend un caractère quasi universel avec 172 membres, l'absence de ce pays, qui compte parmi les initiateurs, paraît incongrue. Pourtant, le gouvernement a signé la convention, et la présidence s'est fortement engagée en sa faveur. De plus, les Etats-Unis financent le Fonds pour l'environnement mondial (FEM), ils satisfont à la plupart des exigences de la convention et ses représentants participent activement, comme observateurs, aux forums internationaux liés à la convention. Mais cette question semble secondaire dans l'agenda politique domestique et les principaux obstacles résident dans l'opposition du Parti républicain et de certains lobbies industriels, pour des raisons économiques. En conséquence, bien que les Etats-Unis aient des moyens alternatifs de se faire entendre, ils ont perdu concrètement l'occasion d'influencer certaines décisions prises par les parties, concernant par exemple la biosécurité ou les clauses sur la répartition des bénéfices liés à l'utilisation des ressources génétiques.

\section{$\square 4^{e}$ Conférence des parties de la Convention sur la biodiversité}

La Convention sur la diversité biologique constitue l'instrument juridique international le plus ambitieux en ce qui concerne la conservation de la biodiversité, son utilisation et la répartition des bénéfices qui pourraient découler de l'utilisation des ressources génétiques. Ces trois objectifs et les moyens d'y parvenir ont été libellés de manière générale dans le texte de la Convention. Il revient à la Conférence des parties (COP), les Etats membres, d'en interpréter les dispositions et de les concrétiser.

La COP-1 (Nassau, Bahamas, du 28 novembre au 9 décembre 1994) a principalement établi un programme de travail, mis en place un mécanisme d'échange d'informations et créé un organe (le SBSTTA, Subsidiary Body on Scientific, Technical and Technological Advice) chargé de donner un avis sur les questions scientifiques et techniques. La COP-2 (Jakarta, Indonésie, du 6 au 17 novembre 1995) a décidé de l'emplacement du secrétariat de la convention; la ville de Montréal a alors été retenue au détriment en particulier de Genève. La conférence a aussi choisi de retenir le Fonds pour l'environnement mondial (FEM) comme mécanisme de financement intérimaire pour la convention, et entamé les travaux sur un protocole additionnel concernant la biosécurité (sur les risques liés au génie génétique, voir Annuaire 1997, p. 57). La COP-3 (Buenos Aires, du 4 au 15 novembre 1996) a procédé à l'évaluation du mécanisme d'échange d'informations, à la reconduction du FEM comme mécanisme financier intérimaire, ainsi qu'à des travaux sur la biodiversité agricole, les savoirs indigènes et les droits de propriété intellectuelle.

La $4^{\mathrm{e}}$ Conférence des parties de la Convention sur la diversité biologique a eu lieu du 4 au 15 mai 1998 à Bratislava, en Slovaquie. Les principaux thèmes abordés ont été: 1) les écosystèmes, particulièrement la biodiversité aquatique des eaux intérieures; 2 ) les politiques de promotion de la biodiversité, y compris le rôle des connaissances traditionnelles, le partage des bénéfices et la sensibilisation du public; 3) les questions institutionnelles: les relations avec les autres conventions et programmes internationaux, ou encore les progrès réalisés par les différents Etats dans l'application des plans nationaux. 
Une importante réflexion a été menée concernant les liens de la convention avec les divers mécanismes et institutions gouvernant les droits de propriété intellectuelle. Les liens entre commerce, propriété intellectuelle, savoirs indigènes et conservation de la biodiversité sont si étroits qu'il est nécessaire de les discuter de manière approfondie. Or, si l'Organisation mondiale du commerce, l'Organisation mondiale de la propriété intellectuelle, les organes de la Convention sur la diversité biologique et le Programme des Nations Unies pour l'environnement (PNUE) reconnaissent le problème et mènent d'intenses débats à ce sujet, aucun forum commun entre ces organisations n'existe. Une discussion menée au cours du Forum global sur la biodiversité (du $1^{\text {er }}$ au 3 mai 1998, sur les lieux de la COP4), mécanisme patronné par l'Union internationale pour la conservation de la nature et de ses ressources (UICN) et qui réunit périodiquement les ONG et les représentants des nations, a conclu à la nécessité de développer une conférence permanente sur ce thème.

Il faut interpréter cette proposition dans le cadre de la controverse soulevée par la proposition du directeur exécutif du PNUE, selon laquelle son organisation devrait jouer un rôle plus important dans la coordination des divers secrétariats liés aux conventions de Rio et des organisations internationales concernées. Cette proposition, contenue dans un rapport des Nations Unies, impliquait une influence accrue du PNUE sur les priorités et les programmes du Fonds pour l'environnement mondial. Les réactions ont été fortes, en particulier celle du directeur du FEM, M. El-Ashry, qui a signifié une fin de non-recevoir à cette proposition, et celle de diverses délégations nationales qui considèrent le PNUE comme un organe superflu du fait de l'existence des conventions de Rio.

Par ailleurs, en réponse à de nombreuses demandes, un groupe de travail sur les peuples indigènes s'est constitué, avec la participation des Etats et des représentants de certains groupes indigènes. Les travaux du groupe, faisant suite à diverses rencontres, ont été centrés sur la question de la protection des savoirs indigènes, et particulièrement sur les mesures concrètes d'application du fameux article $8 \mathrm{j}$ de la convention, qui impose aux Etats parties de respecter et de préserver les connaissances et les pratiques indigènes, mais aussi de consulter ces populations et de les faire participer aux bénéfices de l'utilisation des ressources biologiques. La participation des représentants indigènes à ce groupe de travail n'est pas allée sans soulever l'opposition de certains Etats, comme le Brésil, qui invoquait un strict respect des règles de l'ONU qui n'allouent de pouvoir représentatif qu'aux Etats. Pour dénoncer ce qu'ils ont perçu comme une tentative de censure, les représentants indigènes se sont présentés, dans un mouvement spectaculaire, à la session suivante du groupe avec du ruban adhésif sur la bouche.

Un autre sujet de controverse a été une demande des ONG d'ouvrir un débat sur ce qui est qualifié de Terminator Technology (technologie «terminatrice» en traduction littérale, mais en fait une référence au titre d'un film de science-fiction particulièrement violent) par l'ONG Rural Advancement Foundation International (RAFI). Il s'agit, en substance, d'un brevet pris par une filiale de la compagnie Monsanto des Etats-Unis pour une technique permettant de stériliser les descendants de certaines semences. Elles ne peuvent donc être utilisées qu'une seule fois, sans possibilité de semer à nouveau. Il s'agit d'une façon de contourner ce qui est appelé le «droit des fermiers», à savoir la possibilité pour les cul- 
tivateurs de réutiliser à des fins personnelles des semences achetées auprès des compagnies productrices. Les conséquences en termes de dépendance des cultivateurs et des profits pouvant être tirés de cette technologie sont importantes. Un atelier de travail ad hoc a donné lieu à une polémique entre les représentants du Département américain de l'agriculture, appuyé par plusieurs délégations, et un groupe de pays en développement et d'ONG. La résolution finale demande à l'organe scientifique de la convention (SBSTTA) d'examiner les conséquences de cette technologie.

La prochaine Conférence des parties aura lieu à Nairobi en 2000 et sera centrée autour des écosystèmes arides et semi-arides, de l'utilisation durable des ressources de la biodiversité, et de l'accès à ces ressources. De plus amples informations concernant l'agenda des rencontres internationales liées à la Convention sont disponibles sur le site Internet http://www.igc.org/bionet

\section{La Suisse à Bratislava}

La délégation suisse a assuré pour la deuxième fois la vice-présidence de la conférence et à ce titre Stefan Schwager, de l'Office fédéral de l'environnement, des forêts et du paysage, a présidé la plupart des séances plénières.

Cette réunion a été l'occasion pour la Suisse de présenter le Rapport national de la Suisse pour la Convention sur la diversité biologique, remplissant ainsi, comme l'ont fait 80 pays, une des obligations liées à son appartenance à cette convention internationale (art. 26). Un stand et une exposition ont permis de présenter ce rapport, réalisé par une équipe de consultants privés, aux délégués présents à Bratislava et de faire connaître les progrès enregistrés en Suisse sur la conservation de la biodiversité. A ce propos, selon un examen approfondi des politiques environnementales de la Suisse par 1'OCDE ${ }^{13}$, des succès importants ont été constatés, en particulier dans les domaines de la protection de l'air, des eaux et du transport. Toutefois, le même rapport précise que la diminution des espaces naturels, par la construction et l'agriculture intensive, et la réduction conséquente de la diversité biologique en constituent les points faibles. Des suggestions de mesures concrètes à prendre accompagnent ce rapport.

\section{SOURCES}

Convention sur la diversité biologique.

Diversity, vol. 14, no. 1 \& 2, 1998.

$E C O$ (revue électronique), contact: Stas Burgiel, bionet@igc.org

Earth Negociation Bulletin, http://www.iisd.ca/linkages/french/vol9.html

OCDE, Examen des performances environnementales. Suisse, Paris, 1998.

OFEFP, Bulletin, $2 / 98$.

OFEFP, divers communiqués de presse, http//:www.admin.ch/buwal.

OFEFP, Rapport national de la Suisse pour la Convention sur la diversité biologique, 1998.

PNUE, Dossier d'information. Bratislava 1998.

Rural Advancement Foundation International, communiqués, http://www.rafi.ca 